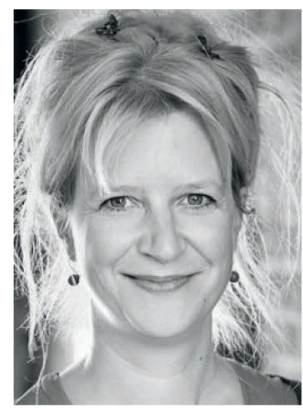

Caroline Weckerle ${ }^{a}$

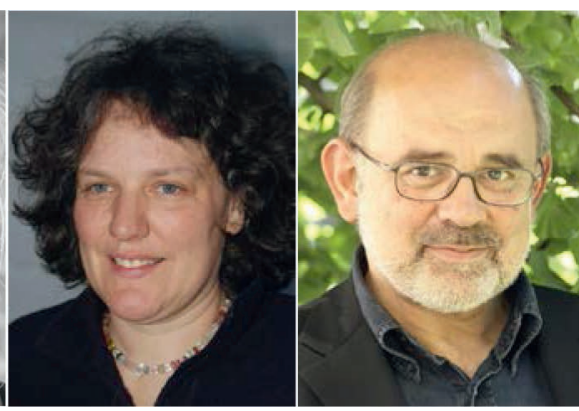

Maja Dal Cero ${ }^{a}$
Reinhard Saller ${ }^{b}$

\section{Pflanzen und Menschen - eine lebenswichtige Beziehung}

Ethnobotanik als Wissenschaft erforscht die Wechselwirkungen zwischen Menschen und Pflanzen. Sie untersucht unter anderem die Verwendung von Medizinalpflanzen und ihre Anwendungen in verschiedenen Kulturen der Welt. Wir Menschen stehen durch unsere Wahrnehmung und unsere Tätigkeiten in permanentem Austausch mit unserer Umwelt, beeinflussen diese und werden von ihr beeinflusst. Die Mensch-Pflanze-Interaktion ist ein Teil davon und liefert der akademischen Forschung eine Vielzahl spannender Fragestellungen: Wer sind die Pflanzenspezialisten in einer Gesellschaft? Welche Pflanzen und welche Pflanzenteile werden zur Arznei- und Heilmittelherstellung verwendet und wie werden diese eingesetzt? Welche Pflanzen spielen im Alltag der Menschen eine wichtige Rolle? Wo werden diese gesammelt? Wie wird die Umwelt genutzt und welchen Einfluss hat diese Nutzung auf die Umwelt? Wenn beispielsweise eine bestimmte chinesische Heilpflanze plötzlich «in Mode kommt» und die Nachfrage nicht nur in China selbst, sondern weltweit wächst, kann es sein, dass in kurzer Zeit ganze Landstriche leergesammelt werden und es Jahrzehnte dauern wird, bis sich die Pflanzenpopulationen wieder erholen können. Ethnobotanik setzt sich also keineswegs nur mit Grundlagenforschung auseinander, sondern untersucht vielmehr auch angewandte Bereiche - wie etwa lokale Arzneipflanzentraditionen.

${ }^{a}$ Institut für Systematische Botanik, Universität Zürich.

bZürich.
Im europäischen Kontext wurde medizinisches Pflanzenwissen seit mehreren Jahrhunderten und zum Teil Jahrtausenden schriftlich festgehalten. Mündliche Traditionen, die Heilpflanzenwissen weitergeben, das nicht auch schriftlich irgendwo zugänglich ist, existieren praktisch nicht mehr. Eine schriftliche Dokumentation allein reicht allerdings nicht aus, um die Bedeutung von Arzneipflanzentraditionen hinreichend zu erfassen oder gar für eine aktuelle Anwendung zu evaluieren. Denn jede Pflanzennutzung ist eine Auseinandersetzung mit einem Lebewesen, das sämtliche Sinne und Emotionen eines Menschen ansprechen und miteinbeziehen kann. Zugleich handelt es sich um soziale Handlungen mit unterschiedlichen Bedeutungen für die verschiedenen Beteiligten, da meist mehrere Personen bei der Verwendung von Heilpflanzen involviert sind. Eine spezielle Kombination von Forschungsmethoden, die den kulturellen Kontext einer Pflanzennutzung beschreiben und interpretieren können, ist also vonnöten.

Ethnobotanik ist eine Disziplin, die vermittelt und verbindet. Sie bedient sich der Methodik und der Theorien aus verschiedenen natur- und geisteswissenschaftlichen Disziplinen und setzt sich mit sogenannten traditionellen Wissenssystemen auseinander. Das heisst, sie bewegt sich an der Berührungsfläche verschiedener Wissenssysteme mit unterschiedlichen Sprachen und Blickwinkeln auf die Realität. Die therapeutische Nutzung traditionellen Pflanzenwissens wird in Europa, und hier vor allem in den deutschsprachigen Ländern, nahezu regelhaft dem weiten Feld der Komplementärmedizin mit ihrer Theorien-, Therapeuten- und Praxisvielfalt zugeordnet. Die enge Verbindung von Komplementärmedizin und Traditioneller Medizin zeigt sich auch in der teilweise synonymen Verwendung beider Begriffe.

Seit 2008 wird an der Universität Zürich ein Zertifikatsstudiengang in Ethnobotanik und Ethnomedizin angeboten. In Zusammenarbeit mit dem Institut für Systematische Botanik und dem Institut für Naturheilkunde des Universitätsspitals Zürich wurde ein interdisziplinäres Angebot entwickelt, das an Menschen gerichtet ist, die sich beruflich oder privat mit der (Heil-)Pflanzennutzung beschäftigen und sich aus unterschiedlichen Blickwinkeln wissenschaftlich damit auseinandersetzen möchten. Die Komplementärmedizin in der Schweiz und in Europa ist dabei genauso ein Thema wie die Medizinsysteme in anderen Weltregionen. Dies ermöglicht, Horizonte zu erweitern und lokale Praktiken in einen globalen Kontext zu setzen. Es zeigt aber auch auf, wie Pflanzenwissen in einer

\section{KARGER}

Fax +497614520714 Information@Karger.com www.karger.com 
globalisierten Welt generiert und weitergegeben wird - ein Thema, das auch in der vielfältigen medizinischen Landschaft der Schweiz eine bedeutende Rolle spielt.

In loser Folge werden in dieser und in den folgenden Ausgaben der SCHWEIZERISCHEN ZEITSCHRIFT FÜR GANZHEITSMEDIZIN Abschlussarbeiten des Studiengangs vorgestellt. Sie werden in gekürzter Form als wissenschaftliche Originalarbeiten bzw. anderweitige wissenschaftliche
Publikationen (z.B. Review, Essay) veröffentlicht. Das übergeordnete Thema lautet «Menschen und Pflanzen», wobei die konkrete Themenwahl mit den beruflichen und/ oder persönlichen Interessen der Absolventen zusammenhängt. Es erwartet Sie also eine bunte und spannende Mischung an Pflanzenthemen, die alle im engeren oder weiteren Sinn mit Ethnobotanik und Heilpflanzen zu tun haben. 